\title{
Comunicação
}

[Communication $]$

\section{Indicadores físico-químicos e atividade antibacteriana de própolis marrom frente à Escherichia coli}

[Physico-chemical indicators and antibacterial activity of brown propolis against Escherichia coli]

\author{
E.M.A.F. Bastos $^{1}$, C. Galbiati $^{2}$, E.M. Loureiro $^{2}$, D.O. Scoaris ${ }^{1}$ \\ ${ }^{1}$ Fundação Ezequiel Dias \\ Rua Conde Pereira Carneiro, 80 \\ 30510-010 - Belo Horizonte, MG \\ ${ }^{2}$ Universidade do Estado de Mato Grosso - Cáceres, MT
}

\begin{abstract}
A busca, na biodiversidade, por recursos genéticos e bioquímicos que possam ser transformados ou contenham moléculas bioativas com potencial atividade terapêutica tem sido alvo de intensas investigações há tempos. Embora essas pesquisas contemplem principalmente a atividade biológica de produtos vegetais, outras substâncias, como a própolis, têm conquistado espaço de relevância.
\end{abstract}

Essa substância, resinosa, é produzida pelas abelhas a partir de exsudatos resinosos de diversas partes das plantas. Esses constituintes são biotransformados pela adição de cera e pela ação da enzima 13-glicosidase, produzida nas glândulas salivares das abelhas, que a depositam nas colmeias, onde tem papel importante para vedar frestas, manter a temperatura interna estável, recobrir o corpo de animais mortos dentro da colmeia e protegê-la contra proliferação de microrganismos (Ghisalberti, 1979).

A composição exata da própolis varia de acordo com a região, a planta fornecedora de resina e a espécie de abelha coletora, refletindo na diversidade de atividades biológicas apresentadas pelo produto (Ghisalberti, 1979). A constituição química básica é uma mistura de substâncias bioativas - ceras, resinas, bálsamos, óleos essenciais e pólen - e outros compostos, como ácido cinâmico, compostos fenólicos e flavonoides, terpenos, ácidos aromáticos,

Recebido em 9 de outubro de 2010

Aceito em 21 de dezembro de 2010

E-mail: embastos@funed.mg.gov.br

Apoio financeiro: FAPEMIG derivados do ácido cafeico, ácidos graxos e aminoácidos. Sua coloração é dependente da origem botânica e pode variar desde a amareloesverdeada, passando por várias tonalidades de marrom até atingir a preta (Bastos, 2001).

Até o momento, inúmeras atividades biológicas já foram atribuídas à própolis: atividade hepatoprotetora, antitumoral, imunomoduladora, regenerativa, cicatrizante, anestésica e antiinflamatória. Mas, reconhecidamente, a atividade biológica mais relevante é a antimicrobiana, sendo atribuída à presença de compostos flavonoides, ácidos fenólicos e seus ésteres (Marcucci et al., 2001). A atividade antibacteriana in vitro já foi evidenciada frente a várias linhagens de bactérias Gram-positivas e negativas (Marcucci et al., 2001; Silici e Kutluca, 2005; Popova et al., 2009), incluindo bactérias anaeróbicas da cavidade oral (Santos et al., 2002) e de interesse veterinário (Bastos et al., 2008), com destaque na inibição de Grampositivos.

Um representante Gram-negativo de grande interesse clínico é a espécie Escherichia coli. Descrita pelo médico austríaco Theodor Escherich, em 1885, este bacilo flagelado, anaeróbico facultativo é membro normal da microbiota gastrointestinal de animais homeotérmicos. No entanto, algumas cepas, denominadas de E. coli diarreiogênicas, são capazes de desencadear infecções intestinais por diferentes mecanismos. Outras cepas podem 
ainda causar infecções extraintestinais, sendo a espécie $E$. coli o principal microrganismo implicado em casos de infecções urinárias e um dos três de maior ocorrência em infecções hospitalares (Tortora et al., 2005).

Pela representatividade clínica, há atualmente uma vasta gama de antimicrobianos destinados ao tratamento de infecções intestinais e extraintestinais causadas por E. coli. Paralelamente, o uso indiscriminado e prolongado destes agentes tem levado à seleção de microrganismos resistentes, tornando a utilização de produtos naturais uma alternativa terapêutica viável. As pesquisas com própolis no Brasil concentram-se em amostras de própolis verde e, até o momento, não há relatos da atividade antibacteriana da própolis marrom frente à $E$. coli.

Pelo exposto, o objetivo deste trabalho foi avaliar a atividade antibacteriana in vitro de extrato de própolis marrom frente à Escherichia coli, relacionando-a aos indicadores de qualidade físico-químicos.

Foram analisadas 23 amostras de própolis marrom, provenientes da região sudoeste do estado de Mato Grosso, Brasil, entre as coordenadas $16^{\circ} 00^{\prime} 02^{\prime \prime} \mathrm{S}$ e $57^{\circ} 39^{\prime} 55^{\prime \prime} \mathrm{W}$ e $16^{\circ} 04^{\prime} 55^{\prime \prime} \mathrm{S}$ e $57^{\circ} 37^{\prime} 25^{\prime \prime} \mathrm{W}$. Nos referidos locais, a própolis foi coletada entre agosto de 2007 e julho de 2008, com coletor de própolis inteligente (CPI). Após a coleta, as amostras foram armazenadas em sacos plásticos com vedação, identificadas e enviadas para análise em laboratório. Todas as amostras foram armazenadas a $-20^{\circ} \mathrm{C}$ até o início das análises laboratoriais.

Os ensaios microbiológicos e físico-químicos foram realizados com extratos etanólicos de própolis. Para a produção destes, alíquotas de $50 \mathrm{~g}$ de própolis bruta foram adicionadas a $100 \mathrm{~mL}$ de álcool etílico $70 \%$ PA, sendo mantidas sob maceração, ao abrigo de luz, por 14 dias. Após este período, as amostras foram filtradas em papel filtro qualitativo e armazenadas em frascos âmbar, à temperatura ambiente. A avaliação dos indicadores de qualidade físicoquímica das amostras de própolis contemplou os seguintes parâmetros: determinação de extrato seco, conteúdo de cera, índice de oxidação e determinação quantitativa de compostos fenólicos e flavonoides, segundo a metodologia descrita por Bastos (2001).

A atividade antibacteriana foi avaliada frente ao microrganismo Escherichia coli ATCC 25922, isolado clínico padrão, proveniente do Laboratório de Microrganismos de Referência do Instituto Nacional de Controle de Qualidade em Saúde - INCQS - FIOCRUZ. A cepa foi mantida em tubos de ágar Mueller Hinton (AMH) inclinado, a $4^{\circ} \mathrm{C}$, com subculturas (repiques) periódicas a cada 15 dias. A cada dois repiques, a cepa foi substituída por uma nova cultura, mantida sob congelamento.

Após a ativação da bactéria em caldo Mueller Hinton, foi preparada uma suspensão a 1-2 × $10^{8}$ unidades formadoras de colônias (UFC)/mL (suspensão padrão), correspondente à turbidez da escala de MacFarland 0,5. Foram realizadas duas diluições seriadas 1:10 para obter, assim a suspensão de trabalho a 1 x $10^{6} \mathrm{UFC} / \mathrm{mL}$. Com auxílio de cotonetes esterilizados, a suspensão foi inoculada nas placas de AMH, em três direções, como padronizado pelo CLSI M2-A8 (Padronização..., 2003), com modificações. Após a completa absorção do inóculo pelo ágar, este foi perfurado com ponteiras de $200 \mathrm{uL}$, estéreis e invertidas, obtendo-se poços de $7 \mathrm{~mm}$. Aos poços, foram adicionados discos de papel (blanck disc), e, sobre estes, inoculados $40 \mu \mathrm{L}$ dos extratos. As placas foram deixadas em repouso até a completa absorção do extrato pelo ágar. Em seguida, foram incubadas de forma invertida, a $37^{\circ} \mathrm{C}$, por 24 horas. $\mathrm{O}$ experimento foi realizado em triplicata, e a atividade antimicrobiana foi considerada positiva quando detectado o desenvolvimento de halo de inibição circundando o poço contendo as diferentes amostras de extrato de própolis. Os halos foram medidos com paquímetro. Como controle, foram empregados discos do antibiótico ampicilina $(10 \mu \mathrm{g}$, Sensifar).

Para determinar a concentração inibitória mínima com potencial atividade antibacteriana, os extratos foram submetidos à concentração em estufa a $40^{\circ} \mathrm{C}$, até que o solvente fosse evaporado. Os extratos concentrados foram ressuspendidos em etanol $70 \%$, à concentração de $1000 \mathrm{mg} / \mathrm{mL}$. A partir da concentração inicial, foram realizadas diluições seriadas 1:2 em etanol $70 \%$, obtendo-se as concentrações de 500, 250, $125,62,5$ e $31,25 \mathrm{mg} / \mathrm{mL}$. As diferentes 
concentrações de cada extrato foram avaliadas frente à cepa teste, como descrito anteriormente, e as amostras foram consideradas ativas quando evidenciados halos de inibição maiores ou iguais a $10 \mathrm{~mm}$.

A composição química da própolis, influenciada pela localização geográfica, pela origem botânica, pelo clima e pela composição do solo, reflete na atividade biológica deste produto natural. A determinação de extrato seco, parâmetro que quantifica a porcentagem de sólidos solúveis extraídos da própolis, apresentou valores entre 2,6 e $27,6 \%$, sendo tanto maior quanto maiores os halos de inibição observados (Tab. 1). Para as amostras ativas, a porcentagem de extrato seco esteve compreendida entre $17,1 \mathrm{e}$ 27,6\%, semelhante aos resultados obtidos para a própolis verde (Bastos, 2001; Pardo, 2007).

Tabela 1. Indicadores de qualidade físico-química e atividade antibacteriana de amostras de própolis do Mato Grosso, MT, frente à Escherichia coli ATCC 25922

\begin{tabular}{|c|c|c|c|c|c|c|}
\hline $\begin{array}{l}\text { Amostras } \\
\text { de } \\
\text { própolis }\end{array}$ & $\begin{array}{l}\text { Porcentagem de } \\
\text { extrato seco } \\
\text { (\%) }\end{array}$ & $\begin{array}{l}\text { Índice de } \\
\text { oxidação } \\
\text { (s) }\end{array}$ & $\begin{array}{c}\text { Porcentagem } \\
\text { de cera } \\
(\%)\end{array}$ & $\begin{array}{l}\text { Índice de } \\
\text { fenólicos } \\
(\mathrm{p} / \mathrm{p})\end{array}$ & $\begin{array}{l}\text { Índice de } \\
\text { flavonoides } \\
(\mathrm{p} / \mathrm{p})\end{array}$ & $\begin{array}{c}\text { Atividade } \\
\text { antibacteriana* } \\
(\mathrm{mm})\end{array}$ \\
\hline 1 & 4,1 & 5'02" & 15,04 & 0,1 & 0,02 & - \\
\hline 2 & 27,58 & $29^{\prime \prime}$ & 29,65 & 0,2 & 0,08 & 11,33 \\
\hline 3 & 4,43 & 6'07" & 15,94 & 0,1 & 0,03 & - \\
\hline 4 & 5,73 & 8'39" & 31,81 & 4,5 & 0,35 & - \\
\hline 5 & 22,02 & $8 "$ & 18,96 & 0,3 & 0,03 & 8,66 \\
\hline 6 & 4,2 & 8'16" & 18,95 & 0,8 & 0,04 & - \\
\hline 7 & 23,01 & $11^{\prime}$ & 4,14 & 3,4 & 0,36 & 11,33 \\
\hline 8 & 3,74 & 5'53" & 24,04 & 0,6 & 0,05 & - \\
\hline 9 & 18,1 & $15^{\prime \prime}$ & 3,42 & 4,9 & 0,4 & 10,33 \\
\hline 10 & 6,87 & 6'36" & 24,75 & 0,4 & 0,03 & - \\
\hline 11 & 19,6702 & 9" & 4,98 & 3,9 & 0,32 & 8 \\
\hline 12 & 2,63 & 5'52" & 32,85 & 0,4 & 0,05 & - \\
\hline 13 & 17,7 & $13^{\prime \prime}$ & 10,18 & 5,0 & 0,66 & 10,66 \\
\hline 14 & 4,93 & 7'09" & 70,07 & 0,2 & 0,02 & - \\
\hline 15 & 17,13 & 6" & 37,91 & 4,4 & 0,61 & 10,66 \\
\hline 16 & 5,75 & 9" & 67,07 & 0,2 & 0,03 & - \\
\hline 17 & 22,51 & 7" & 33,67 & 3,7 & 0,34 & 10 \\
\hline 18 & 3,4 & 8'17" & 68,34 & 0,2 & 0,02 & - \\
\hline 19 & 25,22 & $3 "$ & 17,54 & 4,8 & 0,44 & 11,3 \\
\hline 20 & 2,97 & $5 ' 30^{\prime \prime}$ & 74,58 & 0,4 & 0,04 & - \\
\hline 21 & 26,69 & 3" & 30,22 & 5,5 & 0,52 & 9 \\
\hline 22 & 3,77 & 7'13" & 72,54 & 0,4 & 0,03 & - \\
\hline 23 & 25,8 & 3" & 21,03 & 4,3 & 0,47 & 9,3 \\
\hline Legislação & Mín. 11\% (p/v) & Máx. 22 s & Máx. 1\% ES & Mín. 0,25\% (p/p) & Mín. 0,50\% (p/p) & - \\
\hline
\end{tabular}

Os índices de oxidação observados também apresentaram grande variação, entre três e 519 segundos, sendo este tanto menor quanto maior a porcentagem de extrato seco, exceto para a amostra 16, que, embora tenha apresentado baixo índice de oxidação, apresentou porcentagem de extrato seco de 5,7\%. Para as amostras ativas, os índices observados variaram de três a 29 segundos (Tab. 1), e apenas 14,3\% destas apresentaram índices maiores que o preconizado pela legislação brasileira, que é de 22 segundos (Brasil, 2001). Para a própolis verde, há relação direta entre os índices de oxidação e a quantificação de compostos fenólicos e flavonoides, o que não foi observado para a própolis marrom avaliada neste trabalho, de origem botânica ainda não determinada. $\mathrm{O}$ índice de oxidação da própolis infere sobre a idade e o armazenamento deste produto, sendo tanto maior 
quanto maiores a temperatura e o período de armazenamento (Asis, 1989).

A cera é produzida pelas abelhas e acrescentada à resina coletada das plantas para a produção da própolis. Segundo a legislação vigente, o limite máximo de cera em extrato de própolis é de $1 \%$ do valor de extrato seco. Todas as amostras avaliadas apresentaram porcentagem de cera acima do limite estabelecido, com valores entre 3,4 e $74,6 \%$, sendo possível detectar atividade antibacteriana positiva em amostras com até $37,91 \%$ de cera em sua composição. A alta porcentagem de cera nas amostras de própolis marrom do estado do Mato Grosso é uma característica diferencial entre esta e outras própolis já estudadas (Bastos, 2001).

Na composição química básica da própolis, figuram numerosos compostos fenólicos, principalmente flavonoides e ácidos fenólicos, sendo estes últimos os mais abundantes em amostras de própolis verde (Marcucci et al., 2001). Nas amostras de própolis marrom, os valores de fenólicos e flavonoides estiveram compreendidos entre 0,1 e $5,0(\mathrm{p} / \mathrm{p})$ e 0,02 e 0,66 $(\mathrm{p} / \mathrm{p})$, respectivamente. Uma vez que a atividade antioxidativa da própolis é atribuída aos compostos fenólicos, foi possível observar correlação negativa entre compostos fenólicos e índice de oxidação.

A atividade antimicrobiana da própolis está intimamente relacionada à sua composição química. No presente estudo, das 23 amostras de própolis avaliadas, sete $(30,4 \%)$ apresentaram potencial atividade antibacteriana para Escherichia coli, com halos de inibição entre 10 e $11,3 \mathrm{~mm}$, sendo possível observar que quanto maior o índice de compostos fenólicos, maiores os halos de inibição para as amostras consideradas ativas (Tab. 1). Para as sete amostras ativas, a CIM foi determinada, sendo esta considerada a concentração correspondente a halos de inibição com valores maiores ou iguais a 10,0mm. As concentrações inibitórias mínimas variaram de 125 a $1000 \mathrm{mg} / \mathrm{mL}$. As amostras 2 e 19 apresentaram CIM de $1000 \mathrm{mg} / \mathrm{mL}$; a amostra 9 , de $500 \mathrm{mg} / \mathrm{mL}$; as amostras 13,15 e 17 , de $250 \mathrm{mg} / \mathrm{mL}$ e apenas a amostra 7 apresentou CIM de $125 \mathrm{mg} / \mathrm{mL}$ para $E$. coli. O controle positivo apresentou halos de inibição de $16 \mathrm{~mm}$.

O presente trabalho contribui para o estabelecimento de parâmetros físico-químicos que poderão ser utilizados na regulamentação da própolis marrom produzida no estado do Mato Grosso. A atividade antibacteriana significativa destas amostras frente à Escherichia coli, importante patógeno Gram-negativo, permite indicar uma possível aplicação terapêutica no desenvolvimento de novas formulações para o tratamento de infecções causadas por E. coli.

Palavras-chave: abelha, própolis, atividade antibacteriana, indicadores físico-químicos, Escherichia coli

\begin{abstract}
The activity of 23 samples of ethanolic brown propolis, from the State of Mato Grosso, was investigated against Escherichia coli ATCC 25922. The values of physical and chemical parameters showed significant variation among samples. The percentage of dry extract ranged from 2.6 to $27.6 \%$. The index of oxidation varied from 3 to 519 seconds. All samples showed the percentage of wax higher than the limit preconized by the legislation, with values varying from 3.4 to $74.6 \%$. The quantification of phenolic and flavonoid compounds, responsible for antimicrobial activity, ranged from 0.1 to 5.0 (w/w) and 0.02 to $0.66(w / w)$, respectively, being that the higher the index of phenolic compounds the larger the zones of inhibition. Antibacterial activity was observed in seven out of the 23 samples, demonstrating zones of inhibition ranging from 10 to $11.3 \mathrm{~mm}$. For these active samples, the minimum inhibitory concentration was determined, ranging from 125 to $1000 \mathrm{mg} / \mathrm{mL}$. The value of MIC in $42.9 \%$ of these samples was $250 \mathrm{mg} / \mathrm{mL}$. These results contribute to the establishment of physical and chemical parameters for the regulation of brown propolis and indicate possible therapeutic applicability in the development of formulations for the treatment of infections caused by E. coli.
\end{abstract}

Keywords: bee, brown propolis, antibacterial activity, physico-chemical indicators, Escherichia coli 


\section{AGRADECIMENTO}

Ao Laboratório de Recursos Vegetais e Opoterápicos, Diretoria de Pesquisa e Desenvolvimento, Fundação Ezequiel Dias, Belo Horizonte, MG.

\section{REFERÊNCIAS BIBLIOGRÁFICAS}

ASIS, M. Propoleo, el oro purpura de las abejas. Havana, Cuba: Centro de Información y Documentación Agropecuário, 1989. 255p.

BASTOS, E.M.A.F. Origem botânica $e$ indicadores de qualidade da própolis verde produzida em Minas Gerais. 2001. 152f. Tese (Doutorado) - Universidade de São Paulo, Ribeirão Preto, SP.

BASTOS, E.M.A.F.; SIMONE, M.; JORGE, DM. et al. In vitro study of the antimicrobial activity of Brazilian propolis against Paenibacillus larvae. J. Invertebr. Path., v.97, p.273-281, 2008.

BRASIL. Instrução Normativa n. 3, de 19 de janeiro de 2001, Anexo VII: Regulamento de Identidade e Qualidade de Extrato de Própolis. In: Ministério da Agricultura, Pecuária e Abastecimento, 2001. Disponível em: $<$ http://extranet.agricultura.gov.br/sislegisconsulta/consultarLegislacao. do operacao $=$ visua lizar\&id=1798>. Acesso em 06.10.10.

PADRONIZAÇÃO dos testes de sensibilidade a antimicrobianos por disco-difusão: Norma Aprovada - 8.ed. Brasília, DF: ANVISA, 2003 (NCCL Document M2-A8).
GHISALBERTI, EL. Propolis: a review. Bee World, v.60, p.59-84, 1979.

MARCUCCI, M.C.; FERRERES, F.; GARCÍAVIGUERA, C. et al. Phenolic compounds from Brazillian propolis with pharmacological activities. J. Ethnopharm., v.74, p.105-112, 2001.

PARDO, M.L.A. Comparação entre a atividade antimicrobiana de própolis de abelhas indígenas sem ferrão e de própolis verde de Apis mellifera. 2007. 152f. Dissertação (Mestrado) Universidade Federal de Minas Gerais, Belo Horizonte.

POPOVA, M.P.; CHINOU, J.B.; MAREKOV, I.N. et al. Terpenes with antimicrobial activity from Cretan propolis. Phytochemistry, v.70, p.1262-1271, 2009

SANTOS, F.A.; BASTOS, E.M.A.F.; RODRIGUES, P.H. et al. Susceptibility of Prevotella intermedia/Prevotella nigrescens (and Porphyromonas gingivalis) to propolis (bee glue) and other antimicrobial agents. Anaerobe, v.8, p.9-15, 2002.

SILICI, S.; KUTLUCA, S. Chemical composition and antibacterial activity of propolis collected by three different races of honeybees in the same region. J. Ethnopharmacol., v.99, p.6973, 2005.

TORTORA, G.J.; FUNKE, B.R.; CASE, C.L. et al. Microbiologia. 8.ed. Porto Alegre: Artmed, 2005. 894p. 